\title{
Mass screening for gastric cancer: how to select patients for endoscopic examination
}

\author{
Hirotaka Nakashima $^{1}$, Ryuji Nagahama ${ }^{1}$, Terushige Yamamoto $^{1}$, and Yasuo Ohkura $^{2}$ \\ ${ }^{1}$ Foundation for Detection of Early Gastric Carcinoma, 2-6-12 Kayaba-cho, Nihonbashi, Chuo-ku, Tokyo 103-8790, Japan \\ ${ }^{2}$ Department of Pathology, Kyorin University School of Medicine, Tokyo, Japan
}

\begin{abstract}
Background. Gastroendoscopy (GS) has recently been applied in mass screening for gastric cancer, instead of X-ray examination, to improve the quality of the screening. However, as there are currently limitations in the application of endoscopy in all types of screening for gastric cancer, patients must be selected for mass screening by endoscopy. We investigated how to select individuals for endoscopic examination of gastric cancer screening and the effectiveness of $\mathrm{X}$-ray primary screening before endoscopy.

Methods. All 7942 patients who underwent upper endoscopy for gastric cancer screening at our hospital between April 2005 and March 2008 were divided into two groups: an X-ray screening group (2782 subjects, endoscopy following primary X-ray screening), and a GS direct group (5160 subjects, endoscopy only).

Results. Thirty-seven carcinomas were detected among the 2782 subjects in the $\mathrm{X}$-ray screening group undergoing endoscopy, representing a cancer detection rate of $1.33 \%$. In the GS direct group, 23 carcinomas were detected in the 5160 subjects undergoing endoscopy, representing a cancer detection rate of $0.45 \%$. However, our results suggested that the gastric cancer detection rate by endoscopy was three times higher when it was performed following $X$-ray screening.

Conclusion. There are various conflicting demands for future screening. Our results suggest that it is appropriate to perform an initial X-ray examination followed by endoscopy in prospective screening for gastric cancer.
\end{abstract}

Key words Gastric cancer · Cancer screening - Gastroendoscopy $\cdot$ Photofluorography

\section{Introduction}

In the field of gastrointestinal medicine, advanced endoscopic techniques make more precise diagnosis

Offprints to: $\mathrm{H}$. Nakashima

Received: August 14, 2009 / Accepted: December 3, 2009 and are believed to be more useful than X-ray examinations to detect small gastric cancer lesions. Therefore, endoscopy has recently been applied in mass screening for gastric cancer, instead of X-ray examinations, to improve the quality of screening. However, as there are currently limitations in the application of endoscopy for all types of screening for gastric cancer, such as a lack of workers familiar with the technique and complications associated with endoscopic examinations, patients must be selected for mass screening by endoscopy [1]. We investigated how to select individuals for endoscopic examination of gastric cancer screening and the effectiveness of X-ray primary screening before endoscopy.

\section{Patients and methods}

All 7942 subjects who underwent upper endoscopy for gastric cancer screening at our hospital between April 2005 and March 2008 were divided into two groups: an $\mathrm{X}$-ray screening group (endoscopy following primary X-ray screening: 2782 subjects; mean age, $51.6 \pm 9.4$ years; percentage of males, $82.9 \%$ ) and a GS direct group (endoscopy only: 5160 subjects; mean age, $51.2 \pm$ 9.5 years; percentage of males, $87.2 \%$ ). Our hospital is an institute for gastric cancer detection surveys and our examinations were performed as mass screening for the general working population. No patients are referred to our hospital following a diagnosis of gastric cancer at other hospitals. Choice of the group was at the discretion of the patients, and the authors did not divide the patients into the two groups. X-ray screening examination was performed according to the standard methods recommended by The Japanese Society of Gastroenterological Cancer Screening (Fig. 1) [2]. Among the total of 38971 subjects with X-ray screening for gastric cancer during the study period, the recall rate was $10.5 \%$, and $60.0 \%$ of these recalled subjects underwent 

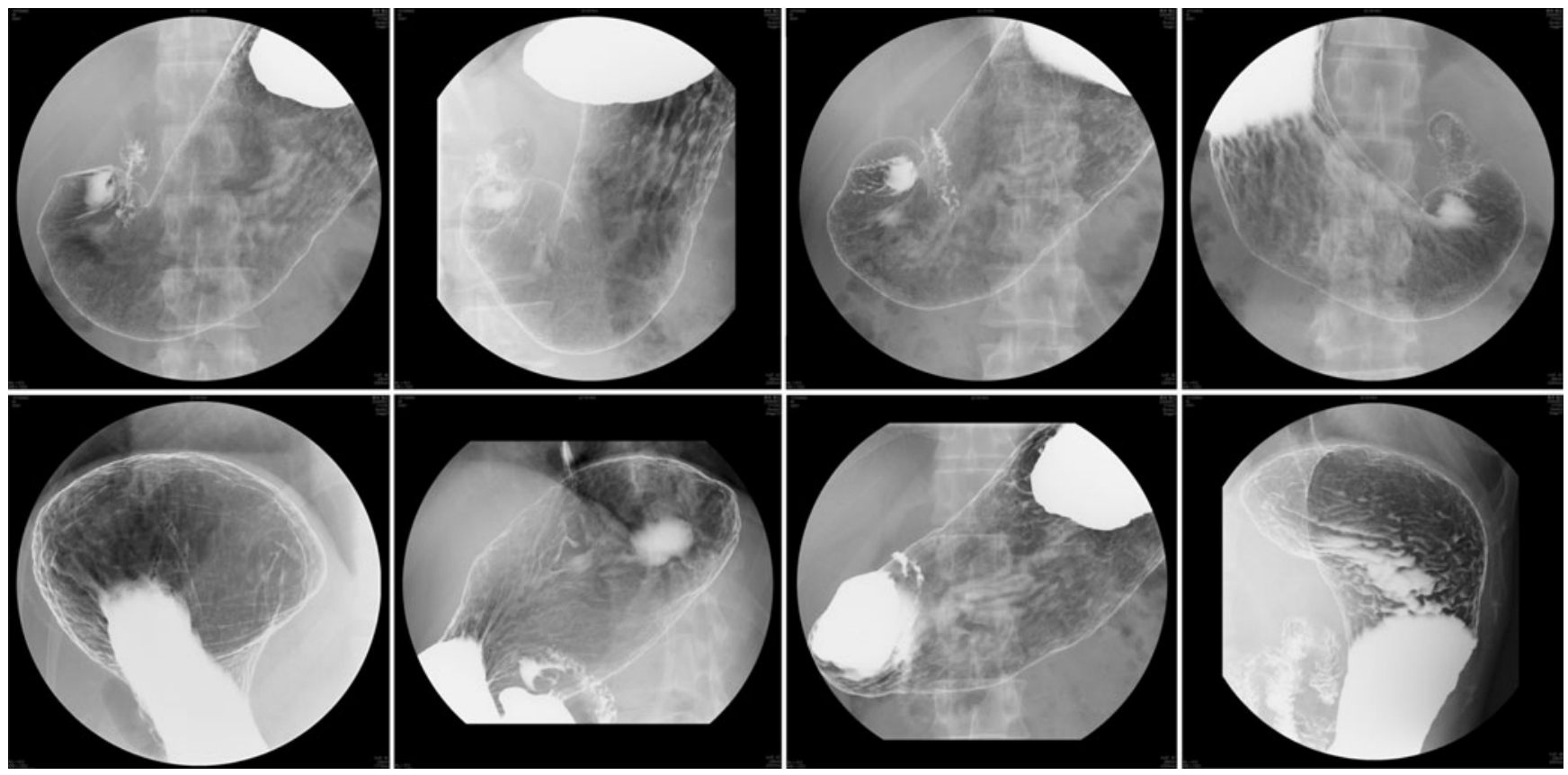

Fig. 1. X-ray screening for gastric cancer, according to the standard methods recommended by The Japanese Society of Gastroenterological Cancer Screening

further examination. (These rates are the averages for 2005, 2006, and 2007.) The esophagus, stomach, and duodenum were inspected by endoscopy regardless of whether X-ray screening was performed, and then biopsies were taken from the lesions at the end of the examination.

The effectiveness of subject selection by X-ray screening was assessed by comparing gastric cancer detection rates by endoscopy between the two groups, i.e., whether fewer endoscopic examinations following X-ray screening could increase the detection rate of gastric cancer compared with that in the GS direct group.

All gastric carcinomas from the two groups were analyzed clinicopathologically to compare the sizes, micro- and macroscopic findings, locations, and depth of invasion. The clinicopathological findings were described according to the Japanese classification of gastric carcinoma [3]. Lesions were classified histopathologically as well-differentiated carcinoma, which included papillary adenocarcinoma (pap) and tubular adenocarcinoma (tub1, tub2), or undifferentiated carcinoma, which included signet-ring cell carcinoma (sig) and poorly differentiated adenocarcinoma (por1, por2).

\section{Results}

Thirty-seven carcinomas were detected among the 2782 subjects in the X-ray screening group undergoing endoscopy, representing a cancer detection rate of $1.33 \%$. In the GS direct group, 23 carcinomas were detected in the 5160 subjects undergoing endoscopy, representing a cancer detection rate of $0.45 \%$. Figure 2 shows the results of gastric cancer detection in the X-ray screening and GS direct groups. The total number of subjects who underwent X-ray screening during the study period was 38971 , and the cancer detection rate was $0.095 \%$. If $100 \%$ of patients were postulated to undergo further examination, the estimated cancer detection rate was $0.16 \%$.

Clinicopathological findings (Table 1) indicated that the lesions found in the GS direct group were smaller and had a higher rate of early-stage cancer than those in the X-ray screening group $(P<0.05, t$-test and $P<$ $0.05, \chi^{2}$ test). Figure 3 shows the locations of the carcinomas in the two groups. In demonstrations of gastric carcinoma in the GS direct group, carcinoma was not noted in the posterior wall of the middle region. Otherwise, in the X-ray screening group some carcinomas were found in the posterior wall of the middle region. In the anterior wall of the upper region, carcinoma was not found in the X-ray screening group or the GS direct group.

In the early gastric cancer patients, the ratios of well-differentiated to undifferentiated carcinoma were 23: 5 and 14: 8 (rates of undifferentiated carcinoma, $17.9 \%$ and $36.4 \%$, respectively) in the X-ray screening group and the GS direct group, respectively. In the advanced cancer patients, the ratio was 4: 5 (rate of undifferentiated carcinoma, 55.6\%) in the X-ray screen- 


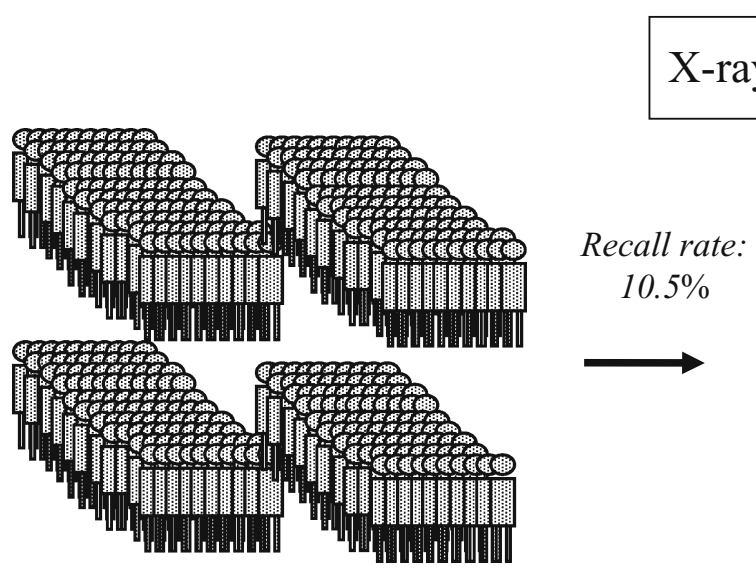

Percentage of subjects

Number undergoing X-ray screening: 38971 individuals who underwent further examination: $60.0 \%$

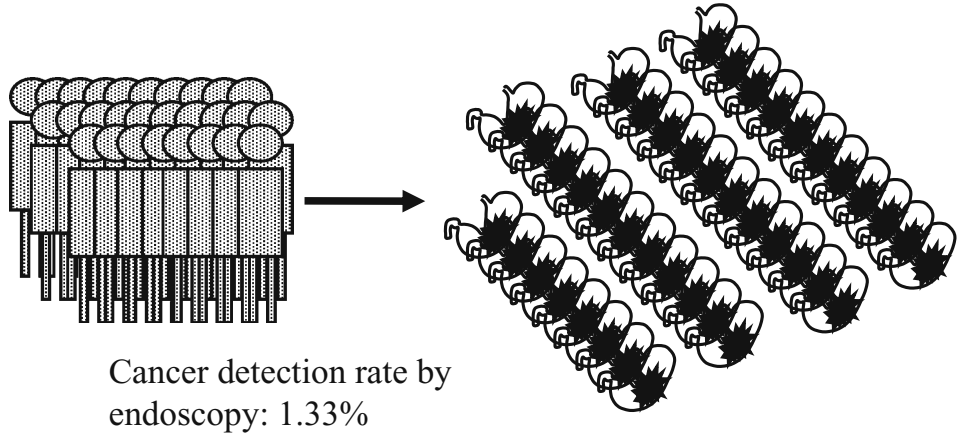

Number of cancers detected: 37 cases

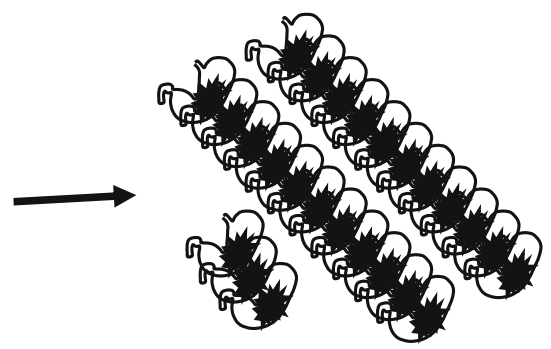

Cancer detection rate: $0.45 \%$

Number of cancers detected: 23 cases

GS direct group: 5160 cases

Fig. 2. Summary of the people undergoing gastric cancer screening

ing group, and there was only 1 case of undifferentiated carcinoma in the GS direct group.

\section{Discussion}

Gastrointestinal endoscopy is widely used because it provides high-resolution images for diagnostic and therapeutic purposes, and it is also a very useful method for discovering small carcinomas. Therefore, there has been some discussion about whether screening by direct gastroendoscopy would be useful to improve the detection and diagnosis of cancers [4]. Diagnostic accuracy for gastric cancer screening is generally evaluated based on the necessity for further examination [(number of patients requiring further examination/number of patients undergoing annual screening) $\times 100$ ], the cancer detection rate [(number of patients diagnosed with cancer/number of patients undergoing annual screening) $\times 100]$, and the rate of early carcinoma [(number of early cancers/number of all cancers $) \times 100$ ] [5].
The purpose of the present study was to investigate whether X-ray examination for the primary screening of gastric cancer would improve the "gastric cancer detection rate" of later endoscopic examination (X-ray screening group) in comparison with that in the GS direct group. Our results suggested that the gastric cancer detection rate by endoscopy was three times higher when it was performed following X-ray screening (Fig. 2). When the clinicopathological findings were compared in the two groups, the lesions found in the GS direct group were smaller and showed a higher rate of early-stage cancer than those in the X-ray screening group (Table 1). The X-ray screening group was selected from a very large mass survey for gastric cancer in about 40000 individuals. We assumed that primary screening by X-ray examination (about 40000 examinations) would effectively identify obvious invasive cancers, which would have a marked influence on the gastric cancer mortality rate. That is, primary screening by X-ray examination (about 40000 examinations) excluded many patients who did not have obvious inva- 
A

O

Early carcinoma well-differentiated type

Advanced carcinoma well-differentiated type

$\triangle$ Early carcinoma undifferentiated type

Post-operative stomach

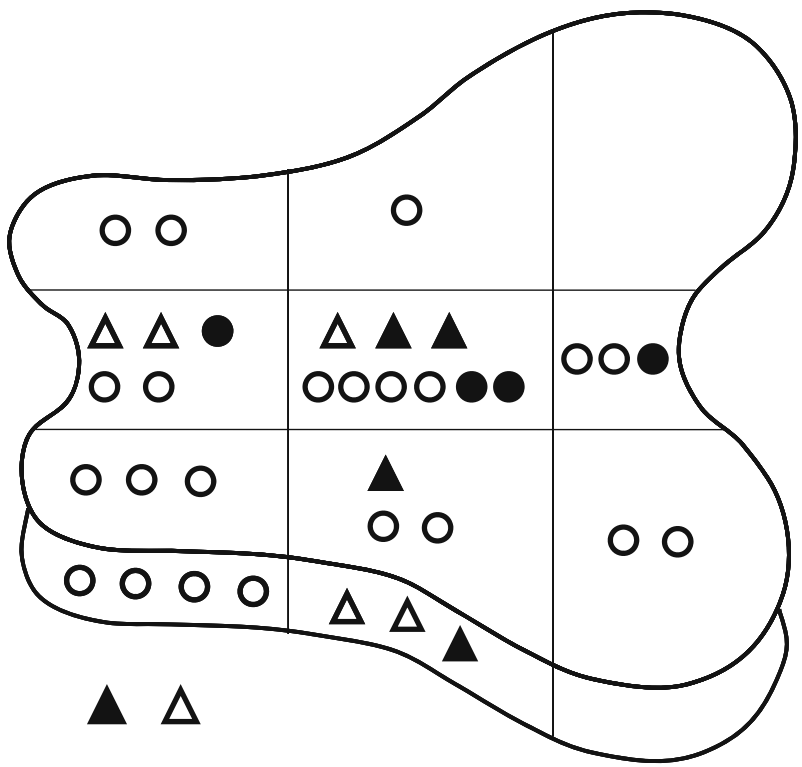

B

Advanced carcinoma undifferentiated type

Early carcinoma well-differentiated type

Advanced carcinoma well-differentiated type

$\Delta$

Early carcinoma undifferentiated type

Advanced carcinoma undifferentiated type

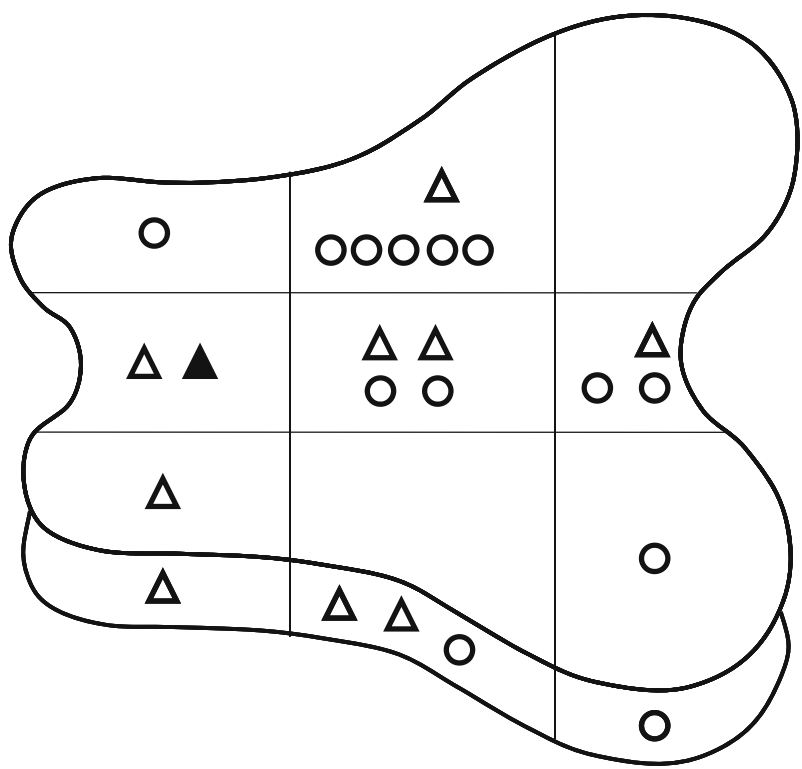

Fig. 3. A Distribution of gastric cancer in the X-ray screening group (37 cases). B Distribution of gastric cancer in the gastroendoscopy (GS) direct group (23 cases)

sive cancer from the group of those patients who required close examination by endoscopy. This was the reason why primary screening by $\mathrm{X}$-ray examination improved the "gastric cancer detection rate" of the endoscopic examination in the "X-ray screening group.

A comparison of lesion locations in the present study indicated that no cancer was detected in the posterior wall of the middle region in the GS direct group, whereas lesions were observed in the posterior wall of the middle region in the X-ray screening group (Fig. 3A, B). These observations confirmed that gastroendoscopy still has limitations due to a blind spot in the posterior wall of the middle region, but this was effectively overcome by $\mathrm{X}$-ray screening, even in the group in which endoscopy was used for definitive diagnosis. On the other hand, fewer cancers were demonstrated in the anterior wall of the middle region in the X-ray screening group compared with the GS direct group. Depiction of the gastric anterior wall by X-ray examination still has some disadvantages, depending on the gastric shape, despite the availability of high-concentration barium techniques and methodological progress. In this area, due to the difficulty of diagnosis by X-ray pictures, careful examination and image interpretation are essential to avoid missing lesions or misdiagnosis.

Well-differentiated intramucosal carcinoma is a good indication for endoscopic treatment. Although intramucosal well-differentiated carcinoma is commonly found at higher rates by endoscopy than by X-ray examination, 16 such lesions were determined by X-ray screen- 
Table 1. Clinicopathological findings

\begin{tabular}{|c|c|c|c|}
\hline Characteristics of carcinoma & X-ray screening group & GS direct group & \\
\hline Number of subjects & $n=37$ & $n=23$ & \\
\hline Mean age (years; mean \pm SD) & $57.3 \pm 11.1$ & $57.2 \pm 7.7$ & \\
\hline Tumor size $(\mathrm{cm} ;$ mean $\pm \mathrm{SD})$ & $2.9 \pm 2.2$ & $1.8 \pm 1.7$ & $(P<0.05 ; t$-test $)$ \\
\hline \multicolumn{4}{|l|}{ Macroscopic type } \\
\hline \multicolumn{4}{|l|}{ Early carcinoma } \\
\hline 0 IIc $\quad 0$ IIc + IIb & 23 & 19 & \\
\hline $0 \mathrm{IIa} \quad 0 \mathrm{IIa}+\mathrm{IIc}$ & 4 & 1 & \\
\hline $0 \mathrm{IIb} \quad 0 \mathrm{IIb}+\mathrm{IIc}$ & 1 & 2 & \\
\hline $0 \mathrm{I}$ & 1 & & \\
\hline \multicolumn{4}{|l|}{ Advanced carcinoma } \\
\hline Type 1 & 1 & & \\
\hline Type 2 or 3 & 6 & 1 & \\
\hline Type 4 & 1 & & \\
\hline \multicolumn{4}{|l|}{ Depth of invasion } \\
\hline $\mathrm{M}$ & 21 & 16 & \\
\hline SM & 7 & 6 & \\
\hline MP & 6 & 1 & \\
\hline $\mathrm{SS} / \mathrm{SE}$ & 3 & 0 & \\
\hline Rate of early carcinoma & $75.7 \%$ & $95.7 \%$ & $\left(P<0.05 ; \chi^{2}\right.$ test $)$ \\
\hline \multicolumn{4}{|l|}{ Histological type } \\
\hline \multicolumn{4}{|l|}{ Early carcinoma } \\
\hline Differentiated type & 23 & 14 & \\
\hline Undifferentiated type & 5 & 8 & \\
\hline \multicolumn{4}{|l|}{ Advanced carcinoma } \\
\hline Differentiated type & 4 & 0 & \\
\hline Undifferentiated type & 5 & 1 & \\
\hline
\end{tabular}

Lesions were classified histopathologically as well-differentiated carcinoma, which included papillary adenocarcinoma (pap) and tubular adenocarcinoma (tub1, tub2), or undifferentiated carcinoma, which included signet-ring cell carcinoma (sig) and poorly differentiated adenocarcinoma (por1, por2)

GS, gastroendoscopy; M, mucosal; SM, submucosal; MP, musclaris propria; SS, subserosa; SE, penetration of serosa

ing in the present study. With regard to therapeutic endoscopy, endoscopic mucosal dissection (ESD) has been widely applied even for large lesions, as long as they are intramucosal and well-differentiated carcinomas [6]. Recently, the rate of identification of gastric cancer has been increased in groups undergoing X-ray screening followed by referral for endoscopic therapy such as ESD.

In the present study, we examined the intervals for gastric cancer screening. To determine screening intervals, the clinicopathological characteristics and prognosis of gastric cancer were investigated. In the X-ray screening group, $24.3 \%$ (9/37 lesions; Table 1) of the subjects had advanced cancer. When we consider natural history of gastric cancer, advanced gastric cancer usually has a poor prognosis after 1 year and this leads to a high mortality rate. Therefore, an interval of 1 year could be reasonable for an X-ray screening group. In the GS direct group, only 1 of the 23 subjects had advanced gastric cancer, which was detected in the first screening. Our previous retrospective study indicated that, over an average follow-up period of 2.4 years in 41 patients with early gastric cancer, 34 lesions $(82.9 \%)$ were intramucosal carcinomas [7]. Advanced cancer is rarely missed because of its obvious macroscopic appearance. A 2-year interval may be suitable for endoscopic screening.

Since the initiation of the screening system for gastric cancer in Japan in the 1960s, X-ray examinations have been performed as the first screening to discover abnormal findings, which were then subjected to endoscopic examination. On the other hand, the selection of highrisk patients for endoscopic examination was attempted using blood tests, serum pepsinogen, and anti-Helicobacter pylori $\mathrm{IgG}$, features that may permit us to control the screening interval by estimating the individual risk of cancer incidence $[8,9]$. However, X-ray examination is a feasible means of evaluating the degree of mucosal atrophy of the whole stomach, according to the distribution of folds in the greater curvature of the gastric body $[10,11]$. We feel that X-ray examination is a useful screening method that yields two important types of information regarding the stomach, allowing the visualization of gastric atrophy and gastric cancer.

Gastrointestinal X-ray diagnosis is better established in Japan than in other countries. There have been many reports of reductions in gastric cancer mortality rates by mass screening for gastric cancer over the 50-year history of these initiatives [12]. Today, there is a great deal of evidence for the effectiveness of X-ray screening 
for gastric cancer in reducing the mortality rate [12]. However, the number of individuals undergoing annual screening appears to have reached a peak. Furthermore, there are various conflicting demands for future screening, e.g., very early stages of cancer should be detected in the screening to avoid the need for more invasive treatment, and simple tests are preferable, such as blood tests, even though they may be inadequate to identify some diseases. Our present results suggest that it is appropriate to perform an initial X-ray examination followed by endoscopy in prospective screening for gastric cancer in Japan, while blood chemistry tests or direct endoscopy, may be a useful alternative.

\section{References}

1. Kozu T, Kakugawa Y, Shoda H, Muramatsu Y, Moriyama N, Saito D. Screening endoscopy for gastric cancer - update of method and difficulties (in Japanese). Clinical Gastroenterology 2008;23:335-44.

2. Guidelines for standard method of stomach radiography (in Japanese). Journal of Gastroenterological Mass Survey 2002;40: 437-47.

3. Japanese classification of gastric carcinoma. - 2nd English edition -. Gastric Cancer 1998;1:10-24.

4. Nishizawa M, Nomoto $K$, Hosoi $T$, Okada $T$, Yamada $K$, Shiga T. Effectiveness of the small-diameter panendoscope in diagnosing cancers of the upper gastrointestinal tract. Endoscopy 1982;14:19-21.

5. Sano H, Hamashima C, Sobue T. Evaluation of process indicators of gastric cancer screening programs among prefectures (in Japanese). Journal of Society for Clinical and Biostatistical Research 2006;26:50-5.

6. Gotoda T, Yanagisawa A, Sasako M, Ono H, Nakanishi Y, Shimoda T, et al. Incidence of lymph node metastasis from early gastric cancer - estimation with a large number of cases at two large centers. Gastric Cancer 2000;3:219-25.

7. Nakashima H, Nagahama R, Ohkura Y, Yamamoto T, Yoshida $\mathrm{T}$, Baba Y, et al. Retrospective study of early gastric cancer followed by endoscopic examination in relation to mucosal back ground (in Japanese). Stomach and Intestine 2008;12:1765-76.

8. Miki K. Endoscopic stomach cancer screening based on the high risk strategy. Gastroenterol Endosc 2007;49:2451-61.

9. Watanabe H, Mitsushima T, Yamaji Y, Okamoto M, Wada R, Kokubo T, et al. Predicting the development of gastric cancer from combining Helicobacter pylori antibodies and serum pepsinogen status: a prospective endoscopic cohort study. Gut 2005;54:764-8.

10. Miyamoto A, Nagahama R, Nakashima H. An evaluation of the degree of gastric mucosal atrophy by $\mathrm{X}$-ray examination and the possibility of the application thereof to gastric cancer screening a comparison with the pepsinogen assay. Journal of Gastroenterological Cancer Screening 2009;47:55-62.

11. Nakashima H, Nagahama R, Yamamoto T. Present state and future of screening X-ray examination for gastric cancer (in Japanese). Gastroenterology (Shoukakika) 2009;49:308-12.

12. Hamashima C, Shibuya D, Yamazaki H, Inoue K, Fukao A, Saito $\mathrm{H}$, et al. The Japanese guidelines for gastric cancer screening. Jpn J Clin Oncol 2008;38:259-67. 